\title{
Quantifying early aftershock activity of the 2004 mid-Niigata Prefecture earthquake $\left(M_{w} 6.6\right)$
}

\author{
Bogdan Enescu, ${ }^{1,2}$ Jim Mori, ${ }^{1}$ and Masatoshi Miyazawa ${ }^{1}$ \\ Received 13 July 2006; revised 17 November 2006; accepted 8 December 2006; published 24 April 2007.
}

[1] We analyze the early aftershock activity of the 2004 mid-Niigata earthquake, using both earthquake catalog data and continuous waveform recordings. The frequencymagnitude distribution analysis of the Japan Meteorological Agency (JMA) catalog shows that the magnitude of completeness of the aftershocks changes from values around 5.0, immediately after the main shock, to about 1.8, 12 hours later. Such a large incompleteness of early events can bias significantly the estimation of aftershock rates. To better determine the temporal pattern of aftershocks in the first minutes after the Niigata earthquake, we analyze the continuous seismograms recorded at six High Sensitivity Seismograph Network (Hi-Net) stations located close to the aftershock distribution. Clear aftershocks can be seen from about $35 \mathrm{~s}$ after the main shock. We estimate that the events we picked on the waveforms recorded at two seismic stations (NGOH and YNTH) situated on opposite sides of the aftershock distribution are complete above a threshold magnitude of 3.4. The $c$ value determined by taking these events into account is about 0.003 days (4.3 min). Statistical tests demonstrate that a small, but nonzero, $c$ value is a reliable result. We also analyze the decay with time of the moment release rates of the aftershocks in the JMA catalog, since these rates should be much less influenced by the missing small events. The moment rates follow a power law time dependence from few minutes to months after the main shock. We finally show that the rate-and-state dependent friction law or stress corrosion could explain well our findings.

Citation: Enescu, B., J. Mori, and M. Miyazawa (2007), Quantifying early aftershock activity of the 2004 mid-Niigata Prefecture earthquake (M $\left.M_{w} 6.6\right)$, J. Geophys. Res., 112, B04310, doi:10.1029/2006JB004629.

\section{Introduction}

[2] The occurrence rate of aftershocks is empirically well described by the modified Omori law [Utsu, 1961]:

$$
n(t)=k /(t+c)^{p},
$$

where $n(t)$ is the frequency of aftershocks per unit time, at time $t$ after the main shock, and $k, c$ and $p$ are constants. The parameter $p$ indicates how fast the rate of aftershocks decays with time and has a value close to 1.0, regardless of the cutoff magnitude. The parameter $k$ is dependent on the total number of events in the sequence. The parameter $c$, which relates to the rate of aftershock activity in the earliest part of an aftershock sequence, typically ranges from 0.5 to 20 hours in empirical studies [Utsu et al., 1995]. Because of deficiencies in recording capabilities, an increased number of smaller early aftershocks are usually missing from seismic catalogs. Kagan [2004] shows that the incompleteness of aftershock data immediately after large main shocks is

\footnotetext{
${ }^{1}$ Earthquake Hazards Division, Disaster Prevention Research Institute, Kyoto University, Kyoto, Japan.

${ }^{2}$ Now at GeoForschungsZentrum, Department 2-Physics of the Earth, Potsdam, Germany.

Copyright 2007 by the American Geophysical Union. 0148-0227/07/2006JB004629\$09.00
}

associated with a systematic bias of the $c$ parameter toward larger values and proposes that $c$ is essentially zero. Other researchers, however, argue that carefully checked catalog data may provide clues on physically based, nonzero $c$ values [e.g., Narteau et al., 2002; Shcherbakov et al., 2004; K. Z. Nanjo et al., The decay of aftershock activity for Japanese earthquakes, submitted to Journal of Geophysical Research, 2006]. Recent work [Vidale et al., 2003; Peng et al., 2006, 2007] brings clear evidence on the increased incompleteness of catalog data immediately after moderate to large earthquakes, while arguing for a deficiency of early aftershocks based on the analysis of high-quality waveform data. As these studies demonstrate, it is essential to accurately determine the $c$ value since the behavior of aftershock sequences during the first minutes and hours after the main shock is a significant component of theoretical models of seismicity [e.g., Dieterich, 1994; Gomberg, 2001; Rubin, 2002]. There is also recent interest in applying stochastic models of earthquake occurrence, employing a power law temporal aftershock decay, to evaluate the time-dependent earthquake probability, as discussed by Kagan [2004]. Therefore, knowing the characteristics of the early part of earthquake sequences is important for obtaining unbiased results.

[3] We focus in this study on the decay of the early aftershock activity following the 2004 mid-Niigata (Chuetsu) earthquake and try to estimate the c parameter by using the Japan Meteorological Agency (JMA) earthquake 


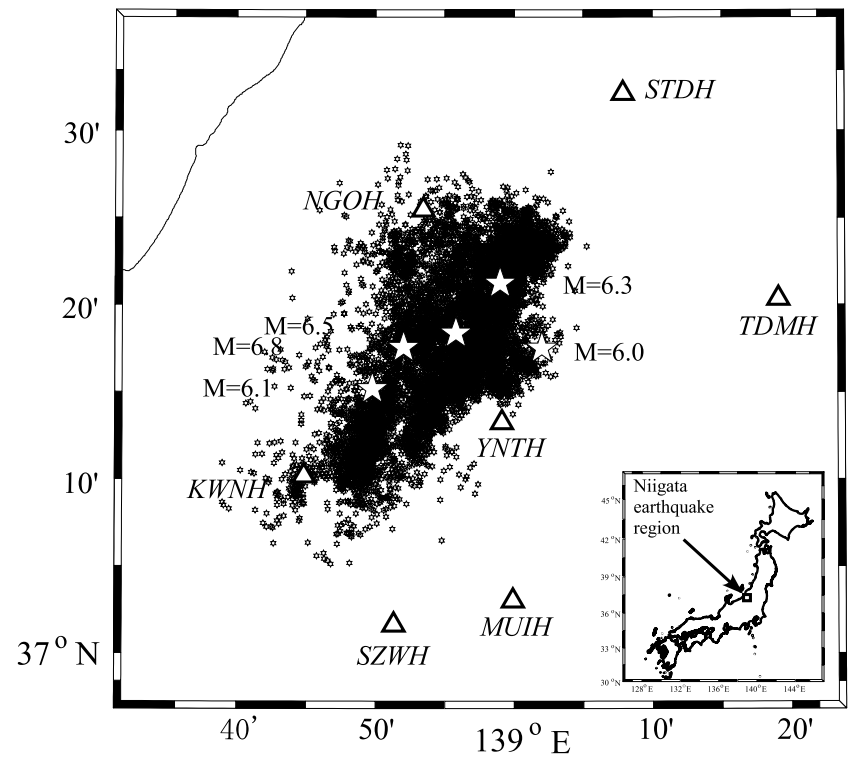

Figure 1. Epicentral map of the 2004 mid-Niigata aftershock sequence (JMA data). Large stars show the locations of the main shock and the larger aftershocks $(M \geq 6.0)$. The locations of the six Hi-Net seismic stations (YNTH, NGOH, MUIH, SZWH, STDH, TDMH) used in section 2.2 are shown by triangles. Inset shows the location of the midNiigata aftershock region in Japan.

catalog data and by counting events identified in the waveform data recorded by Hi-Net, operated by the National Institute for Earth Science and Disaster Prevention (NIED). We also determine the parameter $p$ and the frequencymagnitude distribution of the earthquakes.

\section{Temporal Pattern of Early Aftershocks}

\subsection{JMA Catalog Data}

[4] The map in Figure 1 displays the epicentral distribution of aftershocks (JMA data) for 134 days from the main shock, for $M \geq-0.2$. We chose for analysis all the earthquakes that cluster on and around the fault system in the Chuetsu aftershock region, with depths from 0 to $27 \mathrm{~km}$ [Shibutani et al., 2005]. Few small events, located significantly further from the aftershock cluster in Figure 1, were not included. We also considered a squared aftershock area centered on the mid-Niigata earthquake, with a size defined using the formula of Kagan [2002a] based on the magnitude of the main shock. The obtained data set was essentially the same with the one used in our analysis. The quality of the catalog is high due to the good station coverage in the area, which includes several Hi-Net stations. The main shock (JMA magnitude of $6.8 ; M_{w} 6.6$ ) had four large aftershocks with magnitudes of $6.3,6.0,6.5$ and 6.1 , which occurred $7 \mathrm{~min}, 16 \mathrm{~min}, 38 \mathrm{~min}$, and 88 hours, respectively, after the main shock.

[5] We first analyze the frequency-magnitude distribution of the aftershocks using the JMA catalog with the standard relation of Gutenberg and Richter [1944]:

$$
\log _{10} N=a-b M
$$

where $N$ is the cumulative number of events having magnitude larger than and equal to $M$, and $a$ and $b$ are constants. Figures $2 \mathrm{a}, 2 \mathrm{~b}, 2 \mathrm{c}$, and $2 \mathrm{~d}$ show the frequencymagnitude distribution of earthquakes $0.01,0.05,0.2$, and 0.5 day, respectively after the main shock, for 100 -event windows. We assume that the deviation of the data from a power law fit for small magnitudes is due to the incomplete detection of smaller events. Following a similar procedure to that of Wiemer and Wyss [2000], we determine the magnitude of completeness $(M c)$ as the magnitude at which $95 \%$ of the data can be modeled by a power law fit. The $M c$ value, estimated using the cumulative frequency-magnitude distribution, is also checked against the noncumulative version of the Gutenberg-Richter law. The $b$ value in each case is obtained using a maximum likelihood procedure [Aki, 1965; Utsu, 1965], considering only the earthquakes with magnitudes $M \geq M c$. As Figure 2 clearly shows, $M c$ decreases with time from the main shock. This is consistent with the assumption that there is an improvement with time of the recording capability of small earthquakes. The $b$ values for Figures $2 \mathrm{~b}$ to $2 \mathrm{~d}$ are 0.8 , which are typical values for aftershock sequences [Utsu, 1969]. In Figure $2 \mathrm{a}$, the $b$ value is slightly larger $(0.87)$, but the estimation accuracy might be lower since there are only about 20 events, with magnitudes above $M c$, used for the fit.

[6] Figure 3 shows the magnitude versus time from the main shock for the aftershocks of the JMA catalog. It is clearly seen that as the time from the main shock increases, progressively smaller earthquakes are recorded in the catalog. Thus aftershocks with $M \geq 4.0, M \geq 3.0$ and $M \geq 2.0$ become completely recorded from about 23,114 , and $216 \mathrm{~min}$, respectively, from the main shock. We also plot in Figure 3 the $M c$ values, as determined from the frequency-magnitude distributions (Figure 2). These points fit well (coefficient of determination is 0.99) a least squares regression line with

$$
M c(t)=1.5-1.4 * \log _{10}(t)
$$

where $M c(t)$ is the magnitude of completeness at time $t$ after the main shock. Equation (3) describes the catalog completeness versus time, down to a magnitude of about 1.8. In Figure 3 we plot, for comparison, an average magnitude of completeness line for southern California aftershocks, as determined from equation (15) of Helmstetter et al. [2006] for main shocks with magnitudes $M=6.6$. As can be seen, at very early times after the main shock, even moderate size aftershocks $(M \sim 4.5)$ can be missing from the catalog. This is in agreement with Kagan and Houston [2005], who show that it is very difficult to accurately estimate the $c$ value by analyzing standard catalog data.

\subsection{Hi-Net Waveform Data}

[7] As pointed out in section 2.1, there are probably many small earthquakes missing from the catalog data immediately after the main shock. Therefore we try to detect as many early events as possible on vertical component velocity seismograms recorded at $\mathrm{Hi}-\mathrm{Net}$ borehole stations (Figure 1) situated close to the aftershock distribution. Because of the large amplitudes of the main shock coda waves, it is difficult to accurately identify early aftershocks 

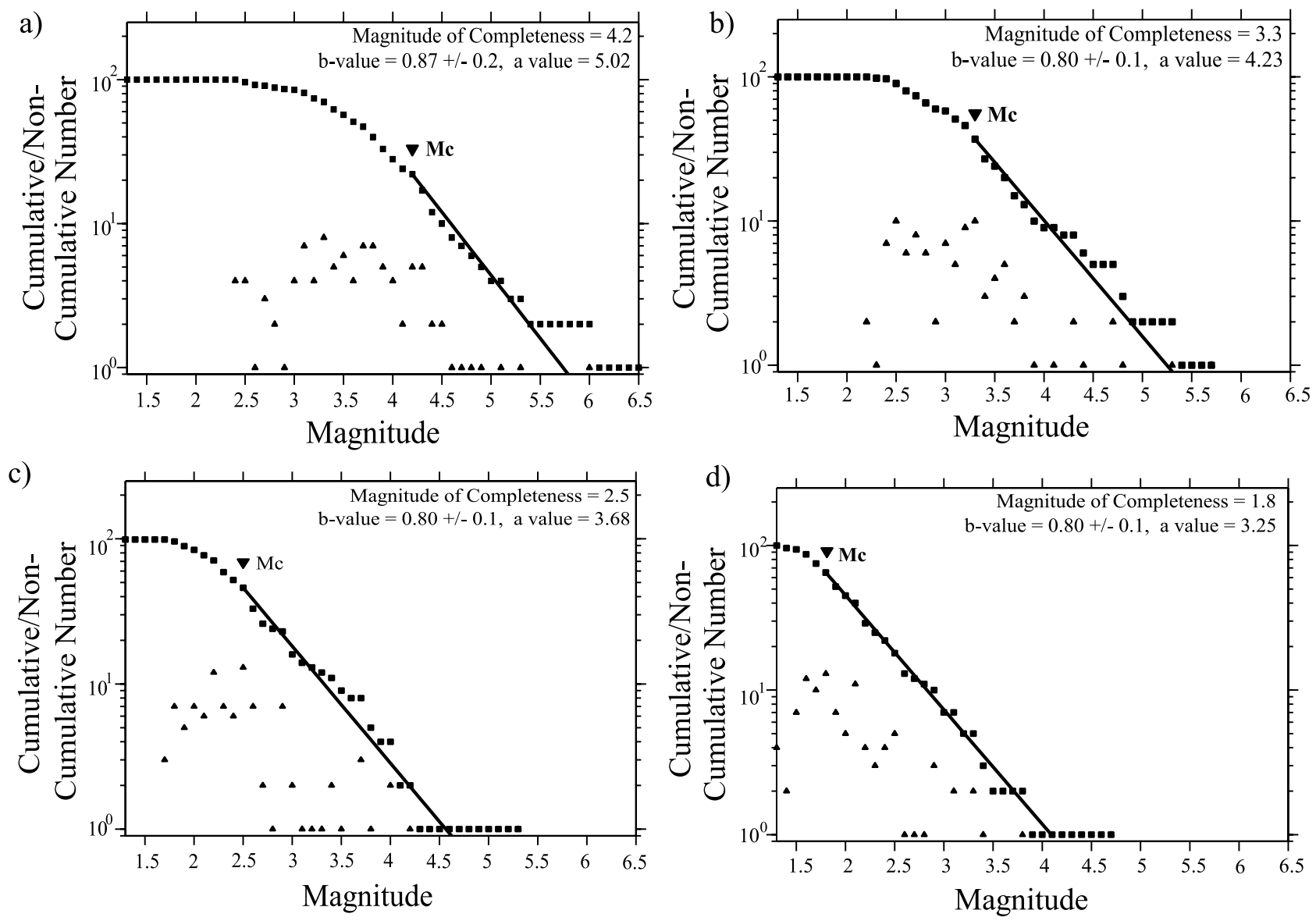

Figure 2. Frequency-magnitude distribution of aftershocks (a) 0.01 day, (b) 0.05 day, (c) 0.2 day and (d) 0.5 day after the main shock, for 100-event windows. The cumulative and noncumulative numbers of earthquakes are shown by rectangles and triangles, respectively. The solid lines represent maximum likelihood fits to the data for magnitudes above the magnitude of completeness, $M c$. The $M c, a$, and $b$ values for each case are also shown.

on the original recordings. Thus, to identify the highfrequency signals from the aftershocks, we high-passfiltered the waveforms. Similar techniques have been used to separate tectonic events from volcanic tremor at times of high volcanic seismicity [e.g., Qamar et al., 1983] or to reveal nuclear tests "hidden" in natural earthquakes [e.g., Sykes, 1986]. We tried several cutoff frequencies: 7, 15, 20, and $25 \mathrm{~Hz}$. Figure 4 shows the results obtained by applying a $7 \mathrm{~Hz}$ high-pass Butterworth filter to the continuous seismograms of six stations. The amplitudes were normalized by the largest value. One can identify clear early aftershocks on the filtered waveforms, starting about $35 \mathrm{~s}$ after the main shock. The results are similar when using higher cutoff frequencies and, in few cases, smaller magnitude events are easier to identify. However, the highfrequency noise is also amplified for higher cutoffs, and this hinders the correct picking of events. Therefore we decided to continue our analysis using the $7 \mathrm{~Hz}$ high-passfiltered seismograms.

[8] We selected two Hi-Net stations (NGOH and YNTH), situated on opposite sides of the aftershock distribution, and count the events with maximum amplitudes above some threshold value, which can be clearly identified on the waveforms. For the $\mathrm{NGOH}$ station we used about $40 \mathrm{~min}$ of continuous data. Unfortunately, station YNTH functioned well for only 5 min after the main shock, so we used the data recorded during this shorter period of time. In several cases, when we were not sure about an event identified at one of the two stations, we also checked the filtered seismograms recorded at the other four stations. For the identified events we cannot determine precisely their absolute occurrence time. However, since both $\mathrm{NGOH}$ and YNTH stations are close to the aftershock region, we approximate their occurrence time with the arrival time, which should be accurate to within a few seconds. To avoid counting both the $P$ and $S$ arrivals from the same event, we checked all picks visually. We finally obtained two data sets of 423 and 65 picks at NGOH and YNTH stations, respectively.

[9] We plot in Figure 5 the cumulative number of picked events at station $\mathrm{NGOH}$ versus the logarithm of their relative, maximum amplitude, measured on rescaled seismograms. For amplitudes above some threshold value the data can be well fit by a straight line with a slope of 1.18 and a standard deviation of 0.03 . In analogy with the frequency-magnitude distribution, the amplitudes $(\geq 0.028)$ that fit the linear relation reflect the completeness level of our picked events. Comparing with located events in the 


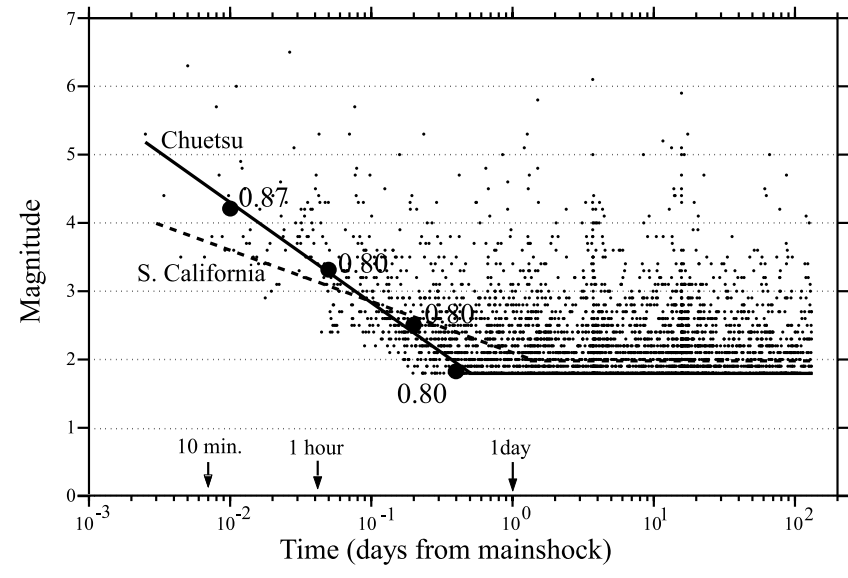

Figure 3. Time from main shock versus magnitude plot for the aftershocks of the 2004 Niigata earthquake $(M \geq 1.8)$. The large dots indicate $M c$ after $0.01,0.05,0.2$, and 0.5 day after the main shock, and the solid line represents a least squares fit to the data. The numbers near each large dot represent the $b$ values for the corresponding relative times from the main shock, determined in Figure 2. The dashed line shows, for comparison, an average magnitude of completeness line for southern California aftershocks, as determined from equation (15) of Helmstetter et al. [2006] for a main shock with magnitude $M=6.6$.
JMA catalog, we find that we are able to identify earthquakes with magnitudes $M \geq 3.4$, which occurred later than about $35 \mathrm{~s}$ after the main shock. There were 41 aftershocks with $M \geq 3.4$ recorded in the JMA catalog in the first $40 \mathrm{~min}$ after the main shock and all of them had relative amplitudes above the 0.028 amplitude threshold. Comparing the amplitudes in our data with the JMA magnitudes for these events, we estimate an uncertainty of about \pm 0.1 for our magnitude determinations. We noticed, however, that the amplitudes of the largest events picked on the filtered waveforms start to saturate for magnitudes larger than about 4.5. Such a saturation may explain why the slope $(1.18 \pm 0.03)$ of the linear fit in Figure 5 is larger than the $b$ values $(\sim 0.8)$ determined previously from the JMA catalog data. The saturation of the larger amplitudes means there is an underestimate of the number of large events (and thus an increase of the slope's value). A similar analysis was performed for the YNTH station, where we could also identify events above a threshold magnitude of 3.4. Most of the events above this threshold, picked at $\mathrm{NGOH}$ and YNTH stations, were also successfully identified on the continuous seismograms of other Hi-Net stations. We note that the Niigata aftershocks were relatively deep; thus there is probably no significant bias due to locations of events close to the stations.

[10] Figure 6 shows the decay of the Niigata aftershock rates using the numbers of events picked at stations $\mathrm{NGOH}$ and YNTH, combined with the JMA data for later times. We selected only aftershocks with $M \geq 3.4$ to compute the logarithmically binned decay rates. The results of the

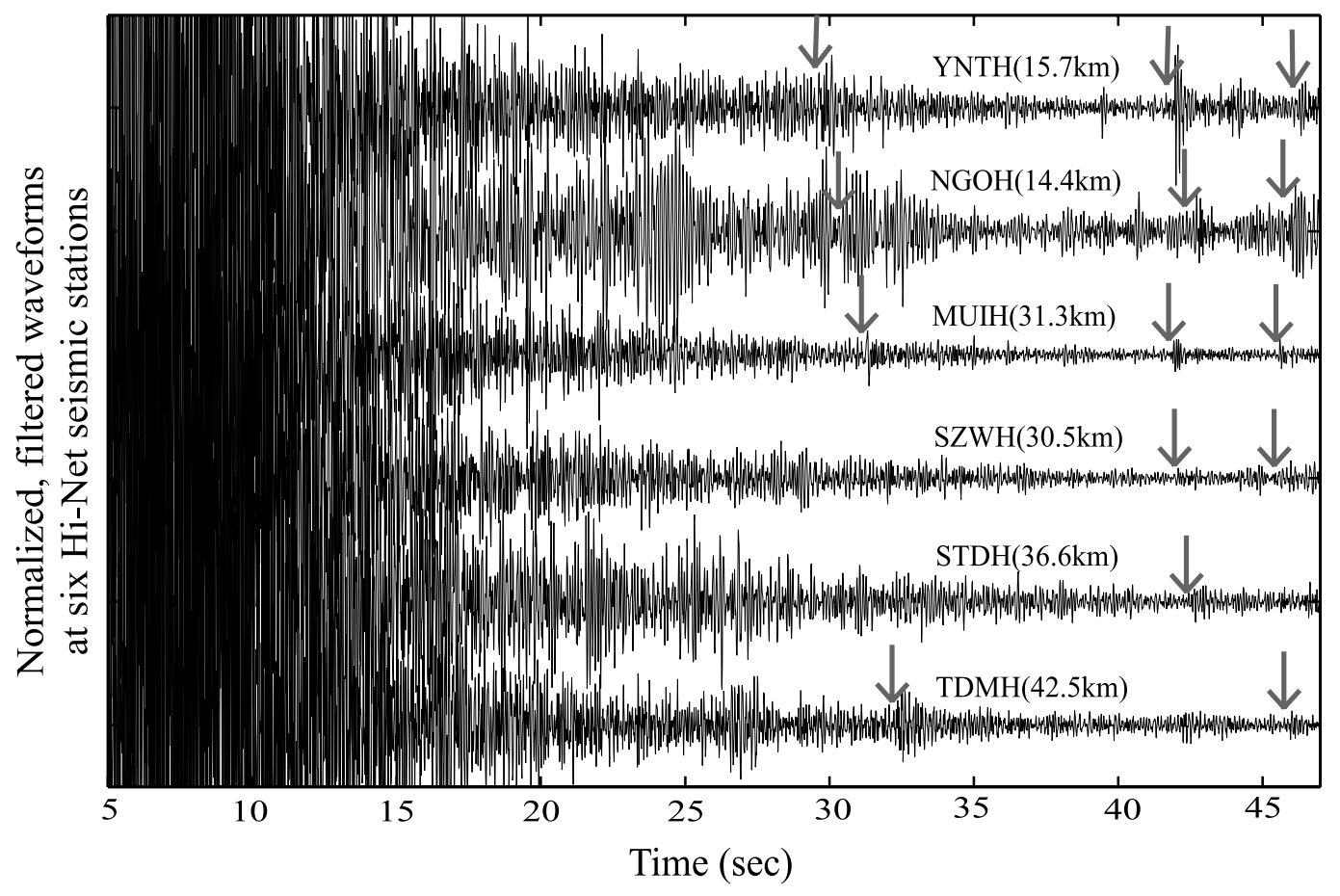

Figure 4. Vertical component velocity seismograms at six Hi-Net stations (see Figure 1), high-pass filtered at $7 \mathrm{~Hz}$ and normalized to the maximum amplitude observed on all seismograms. The names of the recording stations and the corresponding distances to the Niigata main shock are shown. The origin time corresponds to the main shock $P$ wave arrival at YNTH station (1756:3.5 LT, Japanese time). The occurrence time of the main shock is 1756:0.3 LT. The arrows indicate early aftershocks detected on seismograms after filtering. 


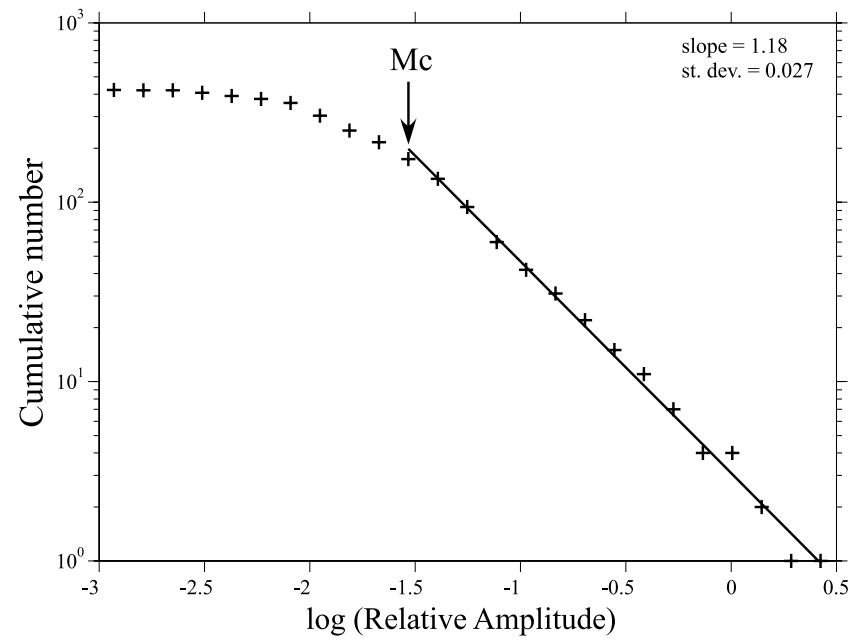

Figure 5. "Frequency-magnitude" like distribution, showing the cumulative number of picked events at $\mathrm{NGOH}$ station versus the logarithm of their relative, maximum amplitude, measured on rescaled seismograms. $M c$ marks the relative amplitude above which our picked event data is complete and corresponds to a magnitude of about 3.4. Because of the high-pass filtering of the waveforms, the magnitudes estimated from the relative amplitudes start saturating around $M=4.5$. The fit of the data above $M c$ has a slope of 1.18 , with a standard deviation of 0.03 . analysis show a $c$ value of 0.003 days $(4.32 \mathrm{~min}$ ). For comparison, the $c$ value determined using only the JMA catalog has a much larger value of 0.017 days $(24.48 \mathrm{~min})$ because of the incompleteness of the data.

[11] The results presented in Figure 6 indicate that the $c$ value of the modified Omori law is relatively small, on the order of just a few minutes. We estimate that the aftershock data sets obtained by picking events on the filtered seismograms are complete for events larger than 3.4 (Figure 5), and that the $c$ value is not a result of incomplete detection of early aftershocks. To help verify our results, we also check how many events we are "missing" that leads to this $c$ value.

[12] The number of aftershocks, $N$, predicted by the modified Omori law (equation (1)) in a certain time interval $\left(t_{1} \leq t<t_{2}\right)$ after the main shock can be easily estimated as

$$
N\left(t_{1}, t_{2}\right)=\int_{t_{1}}^{t_{2}} n(t) d t \equiv \int_{t_{1}}^{t_{2}} \frac{k}{(t+c)^{p}} d t
$$

where $n(t)$ is the aftershock rate and $k, c$ and $p$ are the modified Omori law's parameters. We also know that an aftershock sequence can be described statistically as a nonstationary Poisson process, with the modified Omori law (equation (1)) as intensity function [Ogata, 1983].

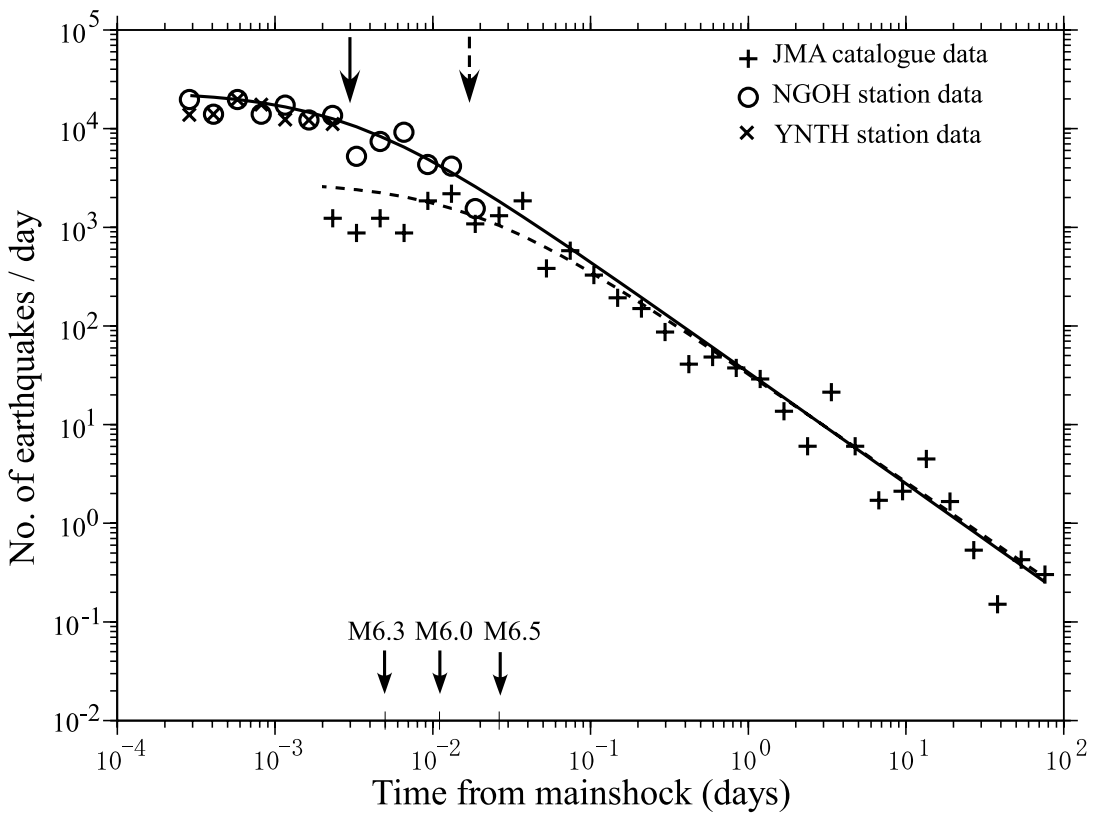

Figure 6. Aftershocks decay rate with time for the JMA catalog data (pluses) and for the events picked on the waveform data at NGOH (circles) and YNTH (crosses) stations. Only events with $M \geq 3.4$ are used. Data were logarithmically binned. The dashed line represents the modified Omori law (equation (1)) fit of the JMA catalog data (287 events) and has the following parameters: $p=1.1 \pm 0.04, c=0.017 \pm$ 0.006 , and $k=33.05 \pm 2.61$. The solid line represents the modified Omori fit for the combined data of JMA catalog and $\mathrm{NGOH}$ station aftershocks (for the overlapping part we used the last source). The fit has the following parameters: $p=1.13 \pm 0.02 ; c=0.003 \pm 0.001$, and $k=33.83 \pm 2.01$. The solid and dotted arrows in the upper part of the figure indicate the $c$ values for the combined data and the JMA data, respectively. 


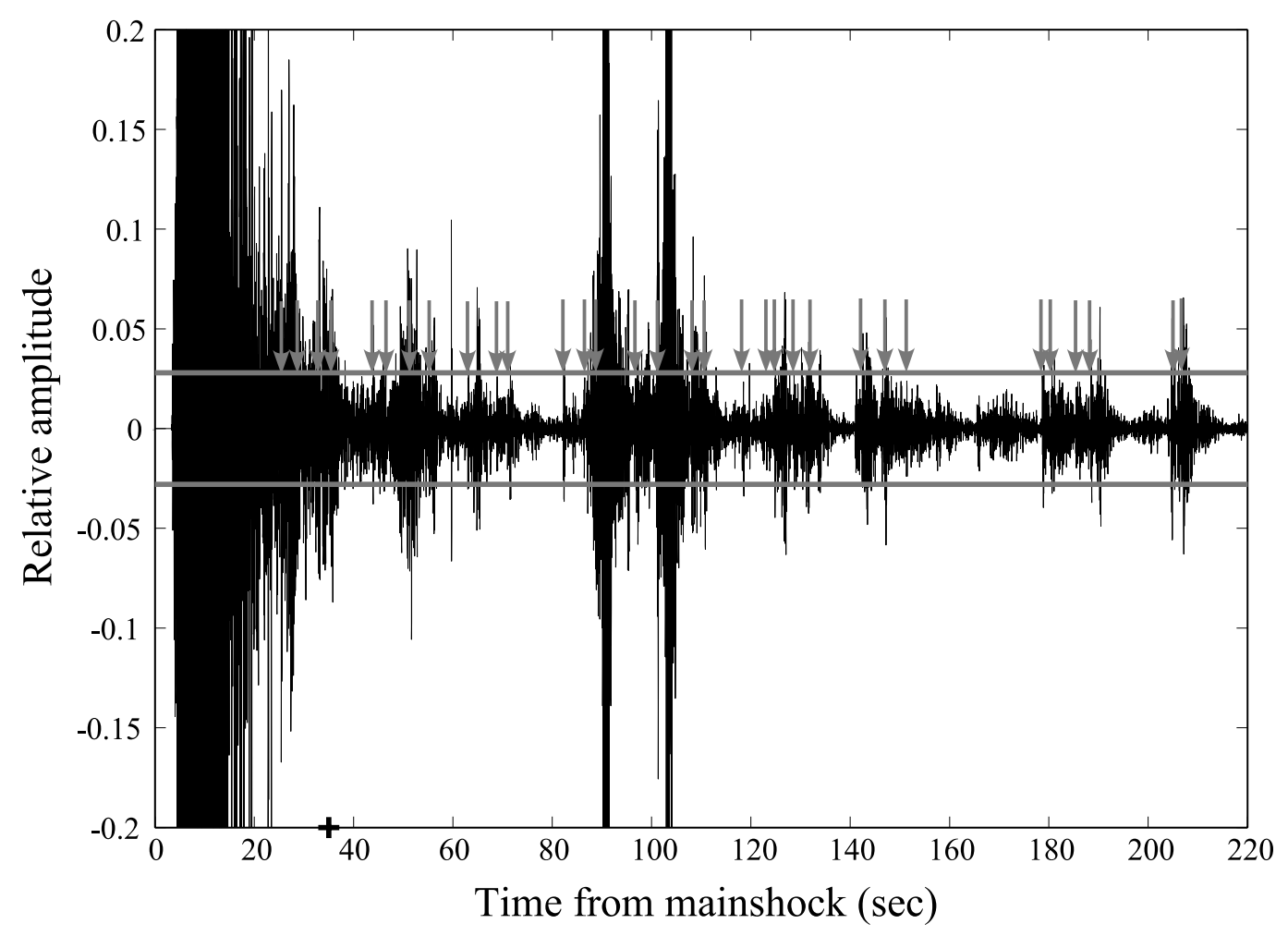

Figure 7. Solid seismogram recorded at $\mathrm{NGOH}$ station, $7 \mathrm{~Hz}$ high-pass filtered and rescaled. The time (s) is relative to the occurrence of the main shock (1756:0.3 LT). The arrows mark the arrival times of the aftershocks with absolute values of amplitude larger than 0.028 . The \pm 0.028 thresholds are indicated by horizontal gray lines. The pluses on the abscissa indicate $35 \mathrm{~s}$ after the main shock.

Then, the probability $P(x)$ of exactly $x$ events occurring in the time range $\left(t_{1} \leq t<t_{2}\right)$ is [Cinlar, 1975]

$$
P(x)=\frac{\left[\int_{t_{1}}^{t_{2}} n(t) d t\right]^{x} \exp -\int_{t_{1}}^{t_{2}} n(t) d t}{x !}
$$

where $n(t)$ is the intensity function (in our case, the aftershocks rate).

[13] We consider $t_{1}=35 \mathrm{~s}$, since we consider that we could count aftershocks which occurred later than about $35 \mathrm{~s}$ from the main shock. We take $t_{2}=220 \mathrm{~s}$, which is slightly smaller than the $P$ wave arrival time of a larger aftershock (M5.3) at the two seismic stations. We also take $p=1.13$ and $k=33.83$ as for the modified Omori fit in Figure 6 but assume that $c=0$. Using formula (4), the number of aftershocks predicted to occur between $35 \mathrm{~s}$ and $220 \mathrm{~s}$, from the main shock, is 156 . We show in Figure 7 the continuous seismogram recorded at $\mathrm{NGOH}$ station for a time period of $220 \mathrm{~s}$ from the occurrence of the main shock. The red arrows mark the arrival times of events with absolute amplitude values larger than 0.028 , which we could count on the filtered waveform. There are 29 such events occurring between $35 \mathrm{~s}$ and $220 \mathrm{~s}$. We may have missed several earthquakes, especially immediately after the two larger aftershocks occurring around $100 \mathrm{~s}$ after the main shock, however, from inspection of the seismogram it is difficult to imagine that we missed 127 events. Using (5), we estimate that the probability of observing no more than 29 events for $35 \mathrm{~s} \leq t<220 \mathrm{~s}$ from the main shock is practically zero. Even by assuming that we have missed counting about 20 events, the probability is still extremely small $\left(<10^{-5}\right)$.

[14] We did the same statistical analysis for the time interval $117 \mathrm{~s} \leq t<220 \mathrm{~s}$, in which no large aftershocks have occurred. In this case the number of events predicted by the modified Omori law is 48 , comparing with 14 events which were observed. The difference between the predicted $(c=0)$ and observed number of events is smaller, however, for this time interval it is very likely that we counted all the events larger than the threshold amplitude value. The probability that our aftershock rates satisfy the $c=0$ assumption is again very small $\left(<10^{-5}\right)$.

[15] We have also tested the difference between the predicted number of aftershocks by assuming a range of values for both $p$ and $k$ parameters (with $c=0$ ) and the observed number. Thus values of the $k$ parameter in a rather large range $(33.83 \pm 12)$ produced a significant difference between the two numbers. The same conclusion holds for a $p$ value in the range: $1.13 \pm 0.1$. These tests show that our results are stable and thus are not significantly influenced by the estimation uncertainties of the modified Omori law parameters. Such uncertainties include the standard deviations of the parameters (Figure 6) and other possible errors caused by the simplified assumptions of the modified Omori fit (equation (1)), as discussed at the end of this section.

[16] The events picked on the continuous waveforms may have some estimation uncertainties. However, the observations of the non-power law decay for the rates of the early aftershocks are similar at two stations situated on different 


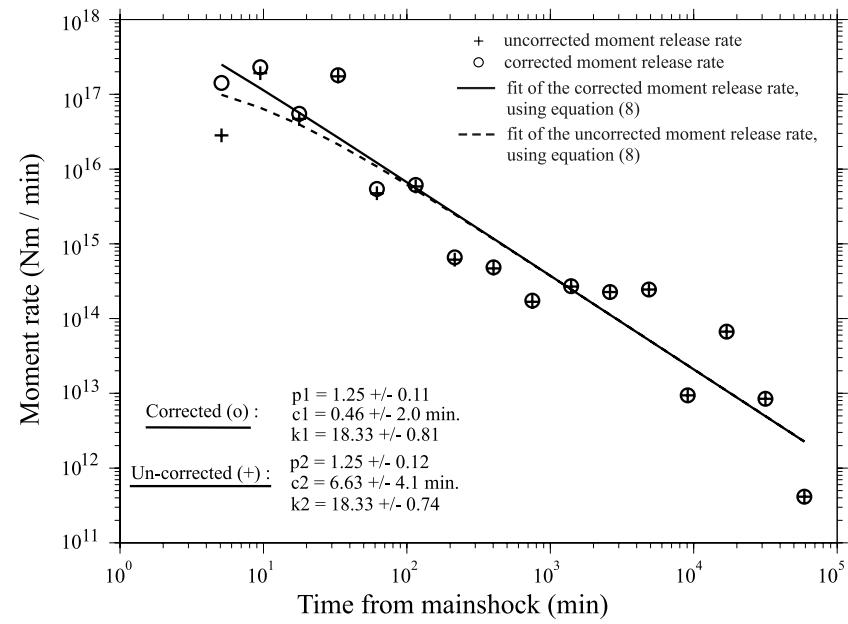

Figure 8. Temporal decay of the uncorrected (crosses) and corrected (circles) moment release rates of aftershocks. The solid and dashed lines are fits of equation (8) to the corrected and uncorrected rates, respectively. The $p 1, c 1$, and $k 1$ parameters characterize the fit of the corrected rates decay, while $p 2, c 2$, and $k 2$ characterize the fit of the uncorrected rates decay.

sides of the earthquake distribution (NGOH and YNTH), suggesting that our results are reliable. We conclude that for the analyzed sequence the $c$ value is small, but has a nonzero value on the order of a few minutes. This result agrees well with a $c$ value of $130 \mathrm{~s}$ obtained by Peng et al. [2006] for the aftershocks of the $2004 M_{w} 6.0$ Parkfield, California, earthquake, by analyzing waveform data from near-source seismometers.

[17] As pointed out by Helmstetter et al. [2005], one of the problems with estimating aftershock properties is the difficulty in distinguishing between the "direct" (triggered by the main shock only) and "secondary" aftershocks (triggered by a previous aftershock). One may address such an issue only by using a stochastic model like ETAS [e.g., Ogata, 1989]. The fits of the aftershocks decay in Figure 6 did not account for the secondary aftershock sequences separately. However, the data fit a modified Omori law well and therefore the determined $p, c$ and $k$ parameters characterize well the temporal decay of this aftershock sequence. The occurrence of M6.3 and M6.0 aftershocks after $7 \mathrm{~min}$ ( 0.005 days) and $16 \mathrm{~min}$ (0.026 days) from the main shock, respectively, probably extended the time periods for which smaller aftershocks are not completely reported in the catalog. This, in turn, makes the determination of the real $c$ values even more difficult. We also note that strong secondary aftershock sequences may produce "bumps" in aftershocks decay rates or the $p$ value estimated from the fit of formula (1) to the data may become large. This is not the case, however, with the Niigata sequence. The $p$ values we determined for several threshold magnitudes are around 1.1, a value similar to the average $p$ values reported in the literature [Utsu et al., 1995; Reasenberg and Jones, 1989]. It was reported [Toda and Kondo, 2005] that the Niigata sequence exhibits a larger number of moderateto-large aftershocks than other similar aftershock sequences.
This translates into relatively higher $k$ values for the modified Omori fit of the Niigata aftershocks decay.

\section{Moment Release of Aftershocks}

[18] The difficulty of estimating real $c$ values from the decay of aftershock rates in seismic catalogs led Kagan and Houston [2005] to an alternative approach. They study the moment release rate of aftershocks as a function of time, which has the advantage that the small events missing in the catalog have much less effect because their seismic moments are relatively small. However, the summation of seismic moments carries a significant price: random fluctuations of the sum are large, because of the small number of summands [Zaliapin et al., 2005; Kagan and Houston, 2005].

[19] From the formula of Takemura [1990], which relates the JMA magnitude, $M$, with the seismic moment $M o\left(\mathrm{~N}^{*} \mathrm{~m}\right)$ :

$$
\log M o=1.17 M+10.72
$$

we calculated $M o$ for each earthquake in the JMA catalog, except the four largest aftershocks of the sequence $(M \geq 6.0)$. For these large events, we used the moments determined by NIED from waveforms inversion.

[20] Assuming that the aftershock size distribution follows the Gutenberg-Richter relation, one can also calculate the moment rate which is due to the missing small aftershocks. To compensate for an incomplete catalog record, we applied a multiplicative correction coefficient to the raw, total seismic moment in an aftershock time interval. The coefficient (C), given by equation (7) of Kagan and Houston [2005], depends on the lower moment threshold, $M_{a}$, of the aftershock sequence and the maximum moment $M_{x p}$ :

$$
C=1-\left(\frac{M_{a}}{M_{x p}}\right)^{1-\beta}
$$

where $\beta=2 b / 3$ [Kagan, 2002b] and $b$ is the $b$ value of the frequency-magnitude distribution.

[21] The threshold moment $\left(M_{a}\right)$ is computed using equations (3) and (6), while $M_{x p}$ is taken as the moment magnitude of the largest aftershock in a certain time period after the main shock, as in the study of Kagan and Houston [2005]. As alternative possibilities, we also equated $M_{x p}$ with the moment of the main shock or to the moment magnitude of the largest aftershock in the sequence. No significant differences between the results were found. We considered $b=0.8$. The results of the moment rate decay are shown in Figure 8.

[22] In Figure 8, there is little difference between the overall moment release rate calculated using the catalog data and the corrected rates, which account for the underreported small aftershocks. However, there is a difference at the very beginning of the aftershock sequence (first time interval). Figure 8 suggests that the aftershock moment rate can be approximated by a modified Omori law time dependence:

$$
M r(t)=k^{\prime} /\left(t+c^{\prime}\right)^{p \prime},
$$




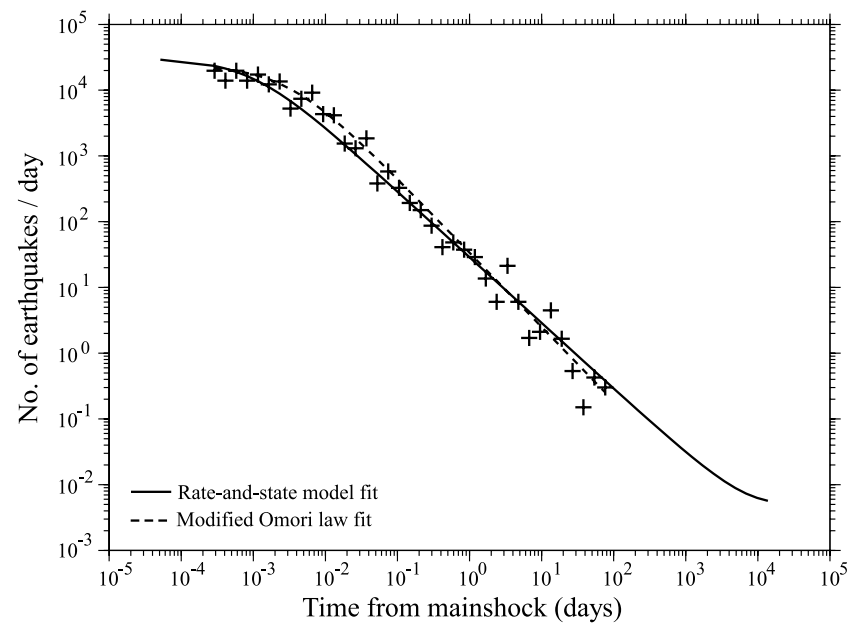

Figure 9. Decay of aftershock activity from the combined data of JMA catalog and the early events picked on filtered waveforms at $\mathrm{NGOH}$ station $(M \geq 3.4)$. The solid and dashed lines represent the data fits of the rate-and-state dependent friction model (equation (9)) and the modified Omori law (equation (1)), respectively. The parameters of the modified Omori law fit are the same as in Figure 6. The parameters of the rate-and-state model fit are discussed in the text.

where $\operatorname{Mr}(t)$ is the moment rate as a function of time, $t$, and $k^{\prime}, c^{\prime}$ and $p^{\prime}$ are constants.

[23] We determined the $k^{\prime}, c^{\prime}$ and $p^{\prime}$ values using a Levenberg-Marquardt nonlinear regression fit for both corrected and original moment data. The $p^{\prime}$ values (around 1.25) are larger than the $p$ values obtained from aftershock rates, which is probably a consequence of weighting much more the larger aftershocks in the computation of moment decay. The $c^{\prime}$ value for the original catalog is $6.6 \mathrm{~min}$ (0.0046 days). This result is obviously independent of any assumption regarding the incompleteness level of the cata$\log$ data and the $b$ value of the aftershocks. The corrected moment rate decay follows a power law. However, there is no moment release in the first 3.6 min after the main shock, as there are no identified aftershocks at these early times in the JMA catalog.

[24] We note that the $c^{\prime}$ estimates are only slightly different from the $c$ value determined from the combined analysis of the JMA catalog and Hi-Net waveform data. However, the uncertainties of the fitting parameters $p^{\prime}$ and $c^{\prime}$ for the decay of both the original and corrected moment release rates (Figure 8) are relatively large compared with those obtained using earthquake numbers (Figure 6).

[25] Since the moment release is dominated by the larger events, the problems of early detection of the catalog data are not as severe, and can be used to describe the general features of the aftershock sequence.

\section{Discussion}

[26] The underlying physics of the temporal decay of aftershocks has attracted much attention from seismologists. Many mechanisms have been proposed, e.g., postseismic creep [e.g., Benioff, 1951], fluid diffusion [Nur and Booker, 1972], rate-and-state dependent friction [Dieterich, 1994], stress corrosion [Yamashita and Knopoff, 1987; Gomberg, 2001] and damage mechanics [Main, 2000; Shcherbakov and Turcotte, 2004; Ben-Zion and Lyakhovsky, 2006]. We focus in this study on the rate-and-state dependent model [Dieterich, 1994], as it has been widely used to explain the temporal pattern of aftershocks.

[27] Dieterich [1994] estimated the seismicity rate as a function of time, $R(t, \tau)$, triggered by a stress change $\tau$ due to the main shock, considering a population of faults governed by the rate-and-state dependent friction law. Assuming a constant tectonic loading rate, $R(t, \tau)$ is given by

$$
R(t, \tau)=\frac{r}{\left(e^{(-\Delta \tau / A \sigma)}-1\right) e^{-t / t_{a}}+1}
$$

where $r$ is background earthquake rate, $A$ is dimensionless fault constitutive parameter; $\sigma$ is normal stress; $\Delta \tau$ is shear stress step due to the main shock and $t_{a}$ is characteristic relaxation time for the perturbation of earthquake rate (aftershock duration). The aftershock duration, $t_{a}$, is:

$$
t_{a}=\frac{A \sigma}{\dot{\tau}}
$$

where $\dot{\tau}$ is the stressing rate, assumed to be the same before and after the main shock. Equation (9) has the following form:

$$
n(t)=k^{\prime \prime} /\left(t+c^{\prime \prime}\right)
$$

with $k^{\prime \prime}$ and $c^{\prime \prime}$ values given by

$$
\begin{gathered}
k^{\prime \prime}=(A \sigma / \dot{\tau}) r \\
c^{\prime \prime}=(A \sigma / \dot{\tau}) \exp \left(\frac{-\Delta \tau}{A \sigma}\right)
\end{gathered}
$$

Equation (11) is a particular case of the modified Omori law (equation (1)) for $p=1$. If $p=1, k^{\prime \prime}=k$ and $c^{\prime \prime}=c$, of the modified Omori law. If $p$ is not equal to one, which is the case of the Niigata sequence, the values are slightly different.

[28] In the case of Niigata earthquake, we estimated the background rate, $r$, by analyzing the seismicity from 1994 to the occurrence time of the 2004 Niigata earthquake, in the aftershock area of the large event. We obtained $r=0.0052$ events/d. This result agrees well with the background rates reported by Toda and Kondo [2005] for several cutoff magnitudes.

[29] Figure 9 shows the decay of aftershock activity of the Niigata earthquake obtained by combining the JMA catalog data with the NGOH station data for the early aftershocks. Only events with $M \geq 3.4$ are used for computing the rates (same as in Figure 6). We use a nonlinear regression technique to fit these data with equation (10), which has two unknown parameters, $t_{a}$ and $\Delta \tau / A \sigma$. The best fit obtained from the inversion is shown as a solid line in Figure 9 and is characterized by $t_{a}=5553.75$ days ( $\sim 15$ years $)$ and $\Delta \tau / A \sigma=15.59$. The coefficient of multiple determination 
for the fit is 0.98 , which indicates that Dieterich's model fits the data well. We also show the fit of the aftershock rates by the modified Omori law (equation (1)). The values of the parameters $p, c$ and $k$ are those indicated for the solid line fit in Figure 6. For most of the data the Dieterich model and the modified Omori law fit the data well.

[30] As mentioned previously, the fit by equation (9) implies a $p$ value of 1.0 for the aftershocks decay. The fit by the modified Omori law, however, shows that the earthquake rates decay slightly faster $(p=1.1)$. This faster decay indicates that the background seismicity level, $r$, will be reached faster and therefore $t_{a}$ would be smaller than predicted by Dieterich's model. Also, the $c$ value estimated by use of equation (13) is 0.001 , smaller than predicted by the modified Omori law. The only way to account for $p>1$ in the rate-and-state model is to assume that the stress $\tau$ decreases with the logarithm of time after the main shock. However, the number of unknown parameters of the model is larger in such a case, so it becomes more difficult to invert for all of them from the aftershock data (the problem becomes less well constrained).

[31] The $A \sigma$ values in the crust are associated with a large range of values. Toda et al. [1998], for example, estimated $A \sigma=0.35$ bars, from the study of aftershocks of the 1995 Kobe earthquake. Assuming this value and the result $\Delta \tau / A \sigma=15.59$, we obtain a stress change $\Delta \tau$ of 5.45 bars. Such a value may be understood as an average over the aftershock region, which includes high values of $\Delta \tau$ close to the main shock rupture and the much smaller values at farther distances from the fault [Miyazawa et al., 2005].

[32] In their review paper, Kanamori and Brodsky [2004] showed that the stress corrosion mechanism [e.g., Das and Scholz, 1981; Gomberg, 2001] predicts a similar behavior for the aftershock decay rates as the rate-and-state dependent friction law, with differences in the long-term return to the background level. In particular, the early aftershock rates deviate from a power law time decay $(\sim 1 / t)$. We also tested this model for the Niigata sequence and obtained a similar fit as for the rate-and-state dependent friction model.

[33] The delayed onset of the power law temporal decay of aftershocks may also be an effect of other phenomena, like strong ground shaking, episodic aseismic slip, healing or fluid diffusion [Vidale et al., 2003; Peng et al., 2007]. Further detailed analysis of high-quality data is necessary to test the predictions of various models and better understand the aftershock generation process.

\section{Conclusions}

[34] The magnitude of completeness of the aftershocks in the JMA catalog changes from values around 5.0, immediately after the Niigata main shock, to about $1.8,12$ hours later. This significant variation mainly reflects the gradual improvement with time of the recording capabilities of smaller earthquakes. The incompleteness of smaller aftershocks in the early part of the sequence introduces a bias in the estimation of the $c$ value. If one uses data where small events are missing, a probably better approach to obtain quick information about the early part of an aftershock sequence is to study the decay of the moment release rate of aftershocks. The delay time $c^{\prime}$ inferred using this technique is 0.0046 days $(6.6 \mathrm{~min})$ for the "original" moment rates (i.e., without any correction).

[35] In order to get more information on the aftershocks in the early part of the Niigata sequence, we analyzed the high-quality waveform data at six Hi-Net stations located close to the aftershock distribution. We counted events on high-pass-filtered waveforms starting from about $35 \mathrm{~s}$ after the main shock. The picked aftershocks are complete above a threshold amplitude that corresponds to a JMA magnitude of 3.4. By analyzing these data together with the JMA catalog of aftershocks, we estimated that the $c$ value is $0.003 \pm 0.001$ days $(4.3 \mathrm{~min})$.

[36] Statistical testing shows that the rates of aftershocks in the first few minutes of the sequence deviate significantly from a power law decay and the rate-and-state dependent friction law or stress corrosion may provide a good physical explanation.

[37] Acknowledgments. We thank NIED and JMA for allowing us to use their earthquake data. B.E. is grateful to the Japan Society for the Promotion of Science (JSPS) for providing him a postdoctoral scholarship to do research at DPRI, Kyoto University. We acknowledge very useful discussions with Hiroo Kanamori and David Jackson. We benefited from the thoughtful comments of the Editor, John C. Mutter, the anonymous Associate Editor and the two reviewers, Zhigang Peng and Ian Main.

\section{References}

Aki, K. (1965), Maximum likelihood estimate of $\mathrm{b}$ in the formula $\log N=$ $a-b M$ and its confidence limits, Bull. Earthquake Res. Inst. Univ. Tokyo, 43, 237-239.

Benioff, H. (1951), Earthquakes and rock creep. Part I: Creep characteristics of rocks and the origin of aftershocks, Bull. Seismol. Soc. Am., 41, $31-62$.

Ben-Zion, Y., and V. Lyakhovsky (2006), Analysis of aftershocks in a lithospheric model with seismogenic zone governed by damage rheology, Geophys. J. Int., 165, 197-210.

Cinlar, E. (1975), Introduction to Stochastic Processes, 432 pp., PrenticeHall, Upper Saddle River, N. J.

Das, S., and C. H. Scholz (1981), Theory of time-dependent rupture in the Earth, J. Geophys. Res., 86, 6039-6051.

Dieterich, J. (1994), A constitutive law for rate of earthquake production and its application to earthquake clustering, J. Geophys. Res., 99, 26012618 .

Gomberg, J. (2001), The failure of earthquake failure models, J. Geophys. Res., 106, 16,253-16,263.

Gutenberg, R., and C. F. Richter (1944), Frequency of earthquakes in California, Bull. Seismol. Soc. Am., 34, 185-188.

Helmstetter, A., Y. Y. Kagan, and D. D. Jackson (2005), Importance of small earthquakes for stress transfers and earthquake triggering, J. Geophys. Res., 110, B05S08, doi:10.1029/2004JB003286.

Helmstetter, A., Y. Y. Kagan, and D. D. Jackson (2006), Comparison of short-term and long-term earthquake forecast models for southern California, Bull. Seismol. Soc. Am., 76, 90-106.

Kagan, Y. Y. (2002a), Aftershock zone scaling, Bull. Seismol. Soc. Am., 92, $641-655$.

Kagan, Y. Y. (2002b), Seismic moment distribution revisited: II. Moment conservation principle, Geophys. J. Int., 149, 731-754.

Kagan, Y. Y. (2004), Short-term properties of earthquake catalogs and models of earthquake source, Bull. Seismol. Soc. Am., 94, 1207-1228.

Kagan, Y. Y., and H. Houston (2005), Relation between mainshock rupture process and Omori's law for aftershock moment release rate, Geophys. J. Int., 163, 1039-1048.

Kanamori, H., and E. Brodsky (2004), The physics of earthquakes, Rep. Prog. Phys., 67, 1429-1496.

Main, I. G. (2000), A damage mechanics model for power-law creep and earthquake aftershock and foreshock sequences, Geophys. J. Int., 142, $151-161$.

Miyazawa, M., J. Mori, Y. Iio, T. Shibutani, S. Matsumoto, H. Katao, S. Ohmi, and K. Nishigami (2005), Triggering sequence of large aftershocks of the Mid Niigata prefecture, Japan earthquake in 2004 by static stress changes, Earth Planets Space, 57, 1109-1113.

Narteau, C., P. Shebalin, and M. Holschneider (2002), Temporal limits of the power law aftershock decay rate, J. Geophys. Res., 107(B12), 2359, doi:10.1029/2002JB001868. 
Nur, A., and J. R. Booker (1972), Aftershocks caused by pore fluid flow?, Science, $175,885-888$

Ogata, Y. (1983), Estimation of the parameters in the modified Omori formula for aftershock frequencies by the maximum likelihood procedure, J. Phys. Earth, 31, 115-124.

Ogata, Y. (1989), Statistical model for standard seismicity and detection of anomalies by residual analysis, Tectonophysics, 169, 159-174.

Peng, Z., J. E. Vidale, and H. Houston (2006), Anomalous early aftershock decay rate of the $2004 M_{w} 6.0$ Parkfield, California, earthquake, Geophys. Res. Lett., 33, L17307, doi:10.1029/2006GL026744.

Peng, Z., J. E. Vidale, M. Ishii, and A. Helmstetter (2007), Seismicity rate immediately before and after main shock rupture from high-frequency waveforms in Japan, J. Geophys. Res., 112, B03306, doi:10.1029/ 2006JB004386.

Qamar, A., W. St. Lawrence, J. N. Moore, and G. Kendrick (1983), Seismic signals preceding the explosive eruption of Mount St. Helens, Washington, on 18 May 1980, Bull. Seismol. Soc. Am., 73, 1797-1813.

Reasenberg, P. A., and L. M. Jones (1989), Earthquake hazard after a mainshock in California, Science, 243, 1173-1176.

Rubin, A. M. (2002), Aftershocks of microearthquakes as probes of the mechanics of rupture, J. Geophys. Res., 107(B7), 2142, doi:10.1029/ 2001JB000496.

Shcherbakov, R., and D. L. Turcotte (2004), A damage mechanics model for aftershocks, Pure Appl. Geophys., 161, 2379-2391.

Shcherbakov, R., D. L. Turcotte, and J. B. Rundle (2004), A generalized Omori's law for earthquake aftershock decay, Geophys. Res. Lett., 31, L11613, doi:10.1029/2004GL019808.

Shibutani, T., et al. (2005), Aftershock distribution of the 2004 mid Niigata Prefecture Earthquake derived from a combined analysis of temporary online observations and permanent observations, Earth Planets Space, $57,357-362$

Sykes, L. R. (1986), Verification of a CTBT, Soviet compliance..., Mod. Geol., 10, 303-321.

Takemura, M. (1990), Magnitude-seismic moment relations for the shallow earthquakes in and around Japan (in Japanese with English abstract), J. Seismol. Soc. Jpn., 43, 257-265.
Toda, S., and K. Kondo (2005), Why the 2004 M6.8 Niigata-ken Chuetsu earthquake produced voluminous aftershocks nearby: an inefficient strain release system, paper presented at Japan Earth and Planetary Science Joint Meeting, Seismol. Soc. of Jpn., Chiba, Japan, 22-26 May.

Toda, S., R. S. Stein, P. A. Reasenberg, J. H. Dieterich, and A. Yoshida (1998), Stress transferred by the $1995 M_{w}=6.9$ Kobe, Japan, shock: Effect on aftershocks and future earthquake probabilities, J. Geophys. Res., 103, 24,543-24,565.

Utsu, T. (1961), A statistical study on the occurrence of aftershocks, Geophys. Mag., 30, 521-605.

Utsu, T. (1965), A method for determining the value of $\mathrm{b}$ in formula $\log N=$ $a-b M$ showing the magnitude-frequency relation for earthquakes (in Japanese), Geophys. Bull. Hokkaido Univ., 13, 99-103.

Utsu, T. (1969), Aftershocks and earthquake statistics (I), J. Fac. Sci., Hokkaido Univ., Ser. VII, 3(3), 129-195.

Utsu, T., Y. Ogata, and R. S. Matsu'ura (1995), The centenary of the Omori formula for a decay law of aftershock activity, J. Phys. Earth, 43, 1-33.

Vidale, J. E., E. S. Cochran, H. Kanamori, and R. W. Clayton (2003), After the lightning and before the thunder: non-Omori behavior of early aftershocks?, Eos Trans. AGU, 84(46), Fall Meet. Suppl., Abstract S31A-08.

Wiemer, S., and M. Wyss (2000), Minimum magnitude of complete reporting in earthquake catalogs: Examples from Alaska, the western United States, and Japan, Bull. Seismol. Soc. Am., 90, 859-869.

Yamashita, T., and L. Knopoff (1987), Models of aftershock occurrence, Geophys. J. R. Astron. Soc., 91, 13-26.

Zaliapin, I. V., Y. Y. Kagan, and F. Schoenberg (2005), Approximating the distribution of Pareto sums, Pure Appl. Geophys., 162, 1187-1228.

B. Enescu, GeoForschungsZentrum, Department 2 "Physics of the Earth", Telegrafenberg E, 456, D-14473, Potsdam, Germany. (benescu@ gfz-potsdam.de)

M. Miyazawa and J. Mori, Earthquake Hazards Division, Disaster Prevention Research Institute, Kyoto University, Gokasho, Uji, Kyoto, 6110011, Japan. 